\title{
Corela
}

Cognition, représentation, langage

10-1 | 2012

Vol. $10, \mathrm{n}^{\circ} 1$

\section{Compte-rendu de : «Les Approches écologiques en linguistique » (N. Lechrevel, 2010) : pour une linguistique « verte »?}

\section{Julien LONGHI}

\section{OpenEdition}

\section{Journals}

Édition électronique

URL : http://journals.openedition.org/corela/2674

DOI : $10.4000 /$ corela. 2674

ISSN : 1638-573X

Éditeur

Cercle linguistique du Centre et de l'Ouest - CerLICO

\section{Référence électronique}

Julien LONGHI, « Compte-rendu de : «Les Approches écologiques en linguistique » (N. Lechrevel, 2010) : pour une linguistique « verte» ?», Corela [En ligne], 10-1 | 2012, mis en ligne le 05 juillet 2012, consulté le 22 septembre 2020. URL : http://journals.openedition.org/corela/2674 ; DOI : https:// doi.org/10.4000/corela.2674

Ce document a été généré automatiquement le 22 septembre 2020.

\section{cc) (i) (2) (2)}

Corela - cognition, représentation, langage est mis à disposition selon les termes de la licence Creative Commons Attribution - Pas d'Utilisation Commerciale - Partage dans les Mêmes Conditions 4.0 International. 


\title{
Compte-rendu de : « Les Approches écologiques en linguistique » (N. Lechrevel, 2010) : pour une linguistique « verte »?
}

\author{
Julien LONGHI
}

\section{RÉFÉRENCE}

Louvain-la-Neuve : Academia Bruylant, coll. « Sciences du langage », EAN :

9782806100047.

1 Le projet de Nadège Lechevrel, à travers une étude sur les approches écologiques en linguistique, participe du décloisonnement opéré depuis quelques années, entre les sciences du vivant et les sciences humaines. Aussi, dans sa préface, Dominique Guillo, souligne que cet ouvrage est précieux et indique que «la référence au vocabulaire de l'écologie en linguistique désigne moins une théorie qu'une méthode ou une approche. En tant que telle, le mérite de cette approche est surtout heuristique, en ce qu'elle invite à assouplir, sinon à abandonner, l'idée selon laquelle la langue serait une structure organisée de façon rigide» (p.13). Pas de dogmatisme donc dans cet ouvrage (même si nous le verrons l'auteure esquisse une préférence pour « un modèle qui synthétise les intuitions et les thèses de l'écolinguistique, de la sociolinguistique, de la cognition située, et de la théorie des affordances de Gibson » (p.14)), mais plutôt une présentation des développements récents des approches écologiques en linguistique, ainsi qu'une nécessaire histoire de ses évolutions. Cet ouvrage offre ainsi un volet pédagogique sur un champ assez peu familier dans le contexte français. C'est pourquoi nous avons mentionné « une linguistique « verte »? » dans notre titre. En effet, si dans de nombreux contextes l'écologie est associée au vert, nous verrons que cet ouvrage 
apporte également des idées nouvelles et rafraichissantes pour l'analyse des langues, et un certain espoir pour la diversité des langues et les langues en danger.

Le premier chapitre entend faire le point sur les modèles écologiques en anthropologie, ethnologie et sociologie: c'est le dualisme nature-culture qui a structuré les débats dans ces champs. Aujourd'hui, l'écologie est traversée par deux courants principaux, l'écologie des populations et l'écologie des écosystèmes. Reprenant l'historique de l'anthropologie et de l'ethnologie, $\mathrm{N}$. Lechevrel indique que les modèles analogiques des années 1950-1960 ont débouché sur des perspectives ultra-fonctionnalistes, et dans les domaines de l'évolution linguistique et de l'acquisition du langage « les perspectives évolutionnistes, les métaphores biologiques et les schémas d'explication naturalistes ont augmenté ces dernières années" (p.30). Les facteurs socioculturels sont souvent « externalisés ». Concernant la sociologie, l'auteure pointe la difficulté de l'écologie humaine issue de la tradition sociologique de Chicago à «se constituer en domaine valide» (p.32) par sa construction en analogie avec une autre discipline : l'écologie humaine se développe cependant très rapidement, tout comme les critiques, avec des débats autour du lien à conserver ou non avec la biologie, des fondements de la zoologie, et de la distinction avec d'autres spécialités. En fait, "derrière ces positionnements se dissimulaient, à l'évidence, de profonds débats et notamment celui du déterminisme biologique "(p.34). Pour en venir à la linguistique écologique, l'auteure relie son analyse à la prise de conscience d'une crise écologique, avec la problématique des langues en danger et la perte de diversité linguistique, allant jusqu'au lien avec la diversité culturelle. L'interdépendance de la diversité culturelle et biologique a aussi fait l'objet de publications, qui corrèlent écosystèmes naturels et indexicalisation de l'environnement dans les langues.

3 Après cette présentation des modèles écologiques dans le champ des sciences humaines, le second chapitre traite plus spécifiquement des approches écologiques en linguistique. Celles-ci connaissent un développement croissant depuis une trentaine d'années, pour l'Europe et les États-Unis, «dans divers domaines des sciences du langage comme la sociolinguistique, la didactique des langues étrangères ou l'évolution et le changement linguistiques " (p.47) sous les noms (pour le français) d'écologie linguistique, linguistique écologique, écologie du langage, écologie des langues, et écolinguistique. L'auteure se demande alors comment aborder cette hétérogénéité sans tomber dans une approche nominaliste. Après une analyse du web, et des sources de publications, l'auteure précise que cette diversité illustre la complexité du domaine. Elle remarque "l'existence de préoccupations communes propres aux domaines du contact des langues, du plurilinguisme et des politiques et planifications linguistiques, conférant au champ de la sociolinguistique un statut privilégié dans l'émergence des approches écologiques » (p.68).

4 Les différents cadres méthodologiques sont les suivants : le changement et l'évolution linguistiques ainsi que la typologie linguistique; les langues en danger et la revitalisation linguistique ; la sociolinguistique ; l'analyse de discours éco-critiques. Ces réceptions sont réparties en deux sous-ensembles: "l'ensemble métaphorique de l'écologie biologique» (p.68) qui convoque des notions comme celles de niche, d'écosystème, de coévolution, ainsi que le vocabulaire relatif aux systèmes complexes et dynamiques; et «l'ensemble métaphorique de l'écologie environnementale » (p.68) qui aura recours aux termes de protection, biodiversité, équilibre et développement 
durable des écosystèmes linguistiques. Dans les sciences cognitives, ces approches concernent les domaines de la cognition située et distribuée.

Le chapitre 3, "Compléter les historiographies ", étudie un élément de la fabrique de l'histoire de la linguistique écologique. Ceci est facilité par l'ouvrage de 2001, de P. Mühlhäusler et A. Fill, The Ecolinguistic Reader. Mais l'ouvrage ne dit pas grand chose, selon l'auteure, de la valeur heuristique et épistémologique, des approches écologiques. Une part importante de ce chapitre est consacrée aux travaux de E. Haugen, de «Langue et immigration » (1938) à "L'écologie du langage » (1971). Ses contributions dans le champ sociolinguistique sont en général connues, et son travail rend compte recherches se limitaient souvent à «la comparaison de traits structurels et [à] la parenté génétique des langues entre elles » (p.77). Il s'est également fait le témoin de l'esprit d'ouverture et d'interdisciplinarité de cette époque. Son texte se présente comme « un projet fédérateur au sein des sciences du langage, dirigé vers l'élaboration interdisciplinaire d'une écologie des langues et du langage» (p.89). Il suggérait une méthode pour décrire les langues de façon totale : rejetant les typologies, il souhaitait voir se généraliser la jonction de plusieurs disciplines, dont l'ethnographie de la communication et la sociolinguistique, l'écologie s'apparentant à une métadiscipline.

6 Le chapitre 4 s'intéresse aux méthodologies en linguistique écologique. L'auteure part des travaux de Haugen, qui se situe dans les recherches dixneuviémistes, et qui visait à dresser un « tableau encyclopédique » des langues du monde. Les travaux de Haarmann (1980, 1986), autour de la notion d'ethnicité, «s'inscrivent dans un courant de recherches plus vaste sur la communication multilingue, tout en renvoyant eux aussi à une démarche écologique, ethnographique ou descriptive» (p.93). Un autre prolongement du texte de Haugen a été proposé par Garner (1986) qui étudie les petites communautés linguistiques d'immigrés : cependant ses conclusions remettent parfois en cause le recours à un modèle écologique.

7 Ces différents travaux s'inscrivent dans l'interdisciplinarité avec d'autres champs des sciences humaines et sociales. D'autres encore suggèrent des échanges avec les sciences naturelles (génétique des populations ou écologie biologique). Pour en rendre compte, l'auteure étudie le domaine de la créolistique en sociolinguistique. Elle détaille en particulier le travail de Mufwene : son modèle d'analyse de restructuration des créoles repose sur des principes de nature plutôt biologique (la spécification, la sélection, la compétition et l'exaptation, et «l'écologie d'une langue est ramenée principalement à l'étude du mouvement des populations qui la parlent »(p.111).

8 La suite de ce chapitre spécifie l'usage de ces méthodologies dans divers domaines. En acquisition par exemple, certains auteurs soutiennent que l'acquisition d'une langue se fait par «boucles dynamiques rétroactives entre pratiques langagières et apprentissages, changement linguistique et perception»(p.120). La linguistique écologique de Lier déplie ces boucles dynamiques, au fondement desquelles se trouvent trois principes : l'activité sémiotique façonne et est façonnée par le langage ; le langage est affordances; l'interaction triadique, ou sémiose inspirée de Peirce, "consiste en une pris en compte des fonctions déictique et indexicale du langage» (p.120). Outre l'acquisition, les politiques linguistiques et les langues en danger sont également concernées par l'écologie des langues. Elle s'attache en effet aux contacts de langues, et s'intéresse au multilinguisme (plutôt qu'au plurilinguisme). Les travaux de L.-J. Calvet s'intègrent dans cette réflexion, dans le but de structurer la pluralité linguistique : les langues se multiplient dans le système écolinguistique, qui est en relation avec le 
milieu. Les recherches de A. Bastardas envisagent quant à elles l'évolution comme coévolution, puisque les organismes évoluent ensemble. Les notions de temps et d'espace se conçoivent en lien avec la notion de continuité.

9 L'auteure propose ensuite une mise en rapport de l'écolinguistique avec les discours environnementaux, dans la lignée des travaux de Mühlhäusler, qui est à la fois sociolinguiste et grammairien générativiste. Il décrit les langues comme «autant de mémoires contenant les capacités des êtres humains à l'inventivité, l'adaptation et la survie. Son but est de montrer que les linguistes ont un rôle à jouer dans la crise écologique, la linguistique pouvant apporter une meilleure connaissance des perceptions de l'environnement à travers l'étude des langues»(p.138). L'approche écologique systémique lui permet de reconstruire la situation linguistique de la région Pacifique avant son exploitation économique par les Européens. Parmi les changements, il recense une diminution de la complexité morphologique et une reconfiguration structurelle et lexico-sémantique convergeant vers les langues européennes. Son approche soutient l'idée d'un développement des grammaires par les usages, conçues comme des grammaires incarnées et processuelles.

10 En remarques conclusives à ce chapitre, l'auteure indique qu'elle aimerait tester une approche écologique qui «se fonderait sur l'idée de langues comme système symbolique » (p.148), l'inspiration écologique se situant au niveau méthodologique, afin d'éviter la confusion vis-à-vis du réalisme qui consiste souvent à substituer l'abstraction à la complexité des phénomènes langagiers.

11 En conclusion, Nadège Lechevrel indique que « la linguistique écologique est surtout à envisager comme une nouvelle façon d'observer et de décrire les langues» (p.151). La linguistique écologique croise de nombreux domaines, et trouve des affinités avec la théorie des systèmes complexes. Nos processus de communication sont capables de faire émerger de nouvelles fonctions cognitives et de nouveaux comportements linguistiques et culturels, conduisant à la notion d'embodiment « comme la nécessité de sortir du paradigme cognitif minimaliste qui réduit le corps au cerveau. Le corps et à la fois le cerveau, le corps physique, et l'interrelation entre l'organisme et son environnement» (p.153).

L'essor d'une linguistique écologique montre également l'intérêt méthodologique et épistémologique du décloisonnement disciplinaire. L'épistémologie interactionniste que soutiennent indirectement ces méthodologies est celle qui défend l'idée que nos représentations sont à la fois informées et informantes de nos interactions. Il faut cependant sortir du «terrain glissant» qui conduit à la critique sur la qualité de l'observation, pour établir une méthodologie en adéquation avec la nature de cet observable à la fois interactionnel et représentationnnel.

13 Cet ouvrage, fort intéressant par les informations qu'il apporte, réussit donc le pari de proposer, à travers les approches écologiques en linguistique, la vision d'une linguistique "verte", qui filerait les diverses acceptions métaphoriques de cette désignation : une linguistique certes liée à l'environnement, mais aussi une linguistique originale et porteuse d'espoir sur les langues comme sur le langage. 


\section{AUTEUR}

\section{JULIEN LONGHI}

Université de Cergy-Pontoise, CRTF 